\title{
ON PHYSICAL \\ MULTIPLE \\ REALIZATION
}

BY

RONALD P. ENDICOTT

Perhaps no argument has done more to set the direction of contemporary philosophy of mind than the "multiple realizability argument." First championed by Hilary Putnam and later reformulated by Jerry Fodor, the argument begins with the observation that a given psychological property can be realized by any number of diverse physical systems, and then ends with the conclusion that psychological properties are irreducible vis-à-vis the physical sciences. ${ }^{1}$ Its target, in other words, is type physicalism and the unity of science, as traditionally conceived.

According to one popular criticism, however, the multiple realizability argument has been discredited by the fact that physical properties are also multiply realized. ${ }^{2}$ But this, I believe, is a mistake. While the criticism does serve to bring a number of important issues into better focus, it can be shown that the facts about physical multiple realization are perfectly consistent with the Putnam-Fodor style argument, and that, when such facts are rightly understood, they do not vitiate the original point about the irreducibility of psychological properties.

\section{Multiple Realization}

In spite of much talk about multiple realization, philosophers have generally been content to leave this central concept unanalyzed. I shall try to remedy this situation by indicating the two most salient features of the concept. First, there is the idea that a property is realized by a number of diverse states, which I take to mean that different state types can provide lawfully sufficient conditions for the instantiation of the multiply realized property. And second, there is the idea that among this range of diverse 
states, there are no lawfully necessary and sufficient conditions for the instantiation of that property. This suggests, then, the following definition, where $\mathrm{M}$ is a multiply realized property, $\mathrm{R}$ the realization base, and $\mathrm{P}$ any property within $\mathrm{R}$ :

$M$ is multiply realized with respect to a range of properties $R$ if and only if (a) $R$ contains some property $P$ which is nomologically sufficient for the instantiation of $M$; and yet (b) $R$ contains no property $P$ which is nomologically necessary and sufficient for the instantiation of $M$.

Clause (a) allows for the existence of one-way conditional laws of the form $(x)(P x=>M x)$, thus giving substance to the claim that it is $P$ in virtue of which an object realizes $M$. Clause (b), however, disallows the existence of any laws of the form ( $\mathrm{x})(\mathrm{Mx}<=>\mathrm{Px})$, thus denying that there are nomological coextensions between $\mathrm{M}$ and any property $\mathrm{P}$ within the multiple realization base. This latter point is the critical one upon which the whole debate has turned, for the crux of the multiple realizability argument consists in the denial of any nomological coextensions between at least some important psychological properties and any physical properties.

So, applying this to the case at hand, let $\mathrm{M}$ be a given psychological property, $\mathrm{R}$ be the entire range of physical properties, and $\mathrm{P}$ any specific property within this range. The claim, then, is that there are important psychological properties for which the concept of multiple realization, so defined, will hold.

One final matter of clarification before we turn to the argument in support of this claim. I shall assume that the psychological properties in question are taken from our best cognitive science, and that the range of physical properteis are taken from a physical or natural science, that is, biology, chemistry, or physics. This is certainly the way matters were understood in the debate over reducing a special or human science to a more basic physical theory, and it also fits nicely with the more traditional controversy about mind/brain identity, points which I shall return to at length.

In any case, the aforementioned claim about multiply realized psychological properties is of great interest since, if true, it would show that a number of traditional philosophical views are mistaken. First and foremost, the doctrine of type physicialism would be in error, since it holds that all mental properties are identical to physical properties, and it is a necessary condition on the identity of properties that they be nomologically coextensive. Second, any view in the philosophy of science according to which psychological theory actually reduces to physical theory by means of biconditional bridge laws would be equally mistaken, at least insofar as the bridge laws are taken to express a genuine nomological connection between the properties.

Now there are roughly three reasons which have been given to accept 
the multiple realizability claim. The first, which I call the appeal to "anomalous realization," is the denial of lawful psychophysical coextensions based upon the allegedly lawless character of any generalization which contains a psychological term. ${ }^{3}$ The second, which I call the appeal to "macro realization," is the denial of lawful psychophysical coextensions based upon an analogy with macro-level types, like being a bridge or a table, which have no uniform microstructures underlying their instances. ${ }^{4}$ And the third, the argument I will briefly canvass here, is the appeal to "functional realization" made famous by Putnam and Fodor. ${ }^{5}$

Hence, according to functionalist theory, and presupposed by standard computational models in psychology, our minds are described in the most theoretically useful way as functional mechanisms, with their mental states being described accordingly as the things which have the appropriate functional roles within this type of system. Thus, a functional property is one defined in terms of such roles, for example, the roles specified by the states of a Turing machine, a method that was once popular; or by a computer program for our cognitive system; or, what is now fashionable, by Ramsey sentences which define psychofunctional properties by their causal role in our best empirical theory of the mind. ${ }^{6}$

But the important point about functional properties, however we understand them, is that they exhibit extreme compositional plasticity in their instantiation. That is, functional properties can be shared by objects of radically different composition and behavior even to the point that they have nothing interesting in common physically. To take a familiar case, two computers with distinct hardware can be functionally isomorphic insofar as they share the functions specified in their programs (or insofar as they satisfy the same Ramsey predicates which define their functional properties). Hence the one may have computer chips made of germanium, while the other may employ the more familiar silicon chips. Or, to dramatize the point, the second may have no chips at all, operating instead by vacuum tubes made of glass and wire, or by punched cards, copper needles, and wheels and pulleys.

The consequence, however, is that there seems to be no chance at all that the same type of physical state realizes the functional properties in each of the two systems. For what physical property could be coextensive (let alone nomologically coextensive) with a functional property shared by the activation of a silicon chip and the movement of a copper needle through a punched card? Consult all the physical sciences, there simply are no plausible candidates.

Since this point has everything to do with functional properties as such, and nothing to do with the specifics of the example, what holds for the functional states of the computer holds for the functional states countenanced in psychology. Hence the above example can be extended to include a functionally isomorphic human being. Or, if we dislike the 
comparison of humans and machines, the same story can be told using only down to earth, flesh and blood creatures. For example, certain mental properties of human beings can remain constant while the neurology differs, especially when damage has occurred to one portion of the brain, and another area has taken over the psychological function. Or it might be that the same psychological states are realized by nonhuman creatures, say, those in another solar system. But given the likely differences in environment, selective pressures, and evolutionary development, it is extremely implausible to think such creatures would have anything like our own neurology.

The net result, as functionalists have emphasized, is that a type of psychological state can be realized in a seemingly infinite number of physically dissimilar ways-by differing neurological structures, extraterrestrial X fibers, silicon chips, pulleys and wheels-as long as these things play the appropriate role within a cognitive system. Yet among this variety of physical structures which are sufficient to realize $M$ there does not seem to be any physical property $P$ which is nomologically necessary and sufficient for $M$, which is precisely the claim about multiply realized psychological properties. Indeed, the logic of the situation seems to be this. Take any $\mathrm{P}$ allegedly coextensive with $\mathrm{M}$. 'The nature of functional properties would seem to guarantee the possibility (a genuine nomic possibility) of a system constructed to realize $M$ without $P$, as in the case of our two computers.

So much, then, for the argument. Let us turn to the criticism.

\section{Multiply Realized Physical Properties}

The criticism can be seperated into two quite different strategies. One requires that we adjust our notion of a property, either by introducing the broader infinite disjunctive properties or the narrower species-specific variety, with the effect that at least some psychophysical laws will be forthcoming. ${ }^{7}$ The other strategy leaves our notion of a property intact, and challenges the very logic of the multiple realizability argument. This latter response is the one we will examine in detail here.

As indicated earlier, this line of response is based upon the observation that physical types and properties are also multiply realized. Jaegwon Kim was the first to suggest that this might create a problem, and he expressed the point in this way:

We of course should not expect to find a physical correlate for every type of mental event we commonly distinguish in daily discourse . . But the situation is hardly peculiar to mental events; we do not expect to find a microphysical basis uniquely correlating with, say, tables either. But this is not to say that tables are not physico-chemical 
structures or that some aspects of tables are not explicable in terms of their microphysical properties. ${ }^{8}$

Hence the common table provides us with a genuine physical type which is multiply realized by distinct microphysical properties. Of course, tables are human artifacts. Hence it is not terribly surprising that the property of being a table lacks a microphysical coextension. But Kim also mentions the case of temperature, noting that:

To argue that the human brain and the canine brain cannot be in the same brain state because of their different physico-chemical structure is like arguing that there can be no microphysical state underlying temperature because all kinds of objects with extremely diverse microphysical compositions can have the same temperature ... difference in material composition with respect to the kind of atoms involved, for example, does not imply difference in the mean kinetic energy of the molecules. ${ }^{9}$

The point again is that temperature is a genuine physical property, one which may even have an underlying microphysical correlate (mean kinetic energy), in spite of the fact that it is realized by various microphysical compositions, in this case, by different types of atoms. The consequence, in Kim's words, is that this fact should "mitigate the sting of Putnam's argument considerably." 10

Donald Davidson has also given a similar argument about multiply realized physical properties, only in this case applied to human action:

It is often said, especially in recent philosophical literature, that there cannot be a physical predicate with the extension of a verb of action . . because there are so many different ways in which an action may be performed. Thus a man may greet a woman by bowing, by saying any number of things, by winking, by whistling; and each of these things may in turn be done in endless ways. The point is fatuous. The particulars that fall under a predicate always differ in endless ways, as long as there are at least two particulars. If the argument were a good one, we could show that acquiring a positive charge is not a physical event, since there are endless ways in which this may happen. ${ }^{11}$

The idea again is that a physical event type or property like acquiring a positive charge remains undeniably physical even though, like a type of action, it can be realized in endless ways. Indeed, Davidson takes the irreducibility argument in question to be "fatuous" precisely because multiple realization is so easily attained by the mental and physical alike.

Finally, Mark Wilson has recently drawn our attention to a number of multiply realized properties in the physical sciences, focusing especially on the case of temperature. ${ }^{12}$ What is interesting here, over and above the facts pointed out by Kim, is the observation that it is not simply that temperature is realized by various microstates, with the correlation between temperature and mean kinetic energy being preserved. Rather, 
that correlation generally fails, and it fails in a way that provides a striking parallel with psychological properties:

If one reexamines the derivation in Ernest Nagel's The Structure of Science which seems to have propelled this particular example into the philosophical limelight, one sees that the mathematics is germane only to a classical gas and won't go through for an arbitrary system. Worse yet, there seems to be no "structural" formula of the expected type which will tell us what temperature should be in all substances. ${ }^{13}$

We are then told that the correlation fails in quantum mechanics for dense gases at low temperatures, and that it fails in Einstein's model for solids, which is just to say that temperature is realized by one thing in ideal gases of a certain range, by another thing in dense gases at low temperatures, by yet another thing in Einsteinian solids, and so on. ${ }^{14}$ But this is very much like saying that a psychological property is realized by one thing in normal humans, by another thing in brain damaged patients, by another thing in extraterrestrials, and the like! Yet, as Wilson emphasizes, in spite of this analogy we are convinced that temperature is a physical property.

Hence, taking all these points together, the sum of the matter seems to be this-since we do not conclude that multiply realized physical properties are nonphysical, then, by parity of reasoning, we cannot conclude that multiply realized psychological properties are nonphysical.

\section{The Real Lesson of Multiple Realization}

But what, exactly, follows from the point that physical properties are multiply realized? Contrary to the above line of response, I think we can happily concede the existence of multiply realized physical properties and still maintain that the original claim has been established, namely, that psychological properties are not lawfully coextensive and hence not identical to physical properties in virtue of being multiply realized by them.

First, remember that a property $\mathrm{M}$ is multiply realized with respect to a range of properties $R$, its realization base, if and only if (a) $R$ contains a property $\mathrm{P}$ which is sufficient for $\mathrm{M}$, and yet (b) $\mathrm{R}$ contains no property $\mathrm{P}$ which is necessary and sufficient for $M$.

Now observe that it is fairly easy for a property to be multiply realized, even a physical one. Consider Davidson's case of acquiring a positive charge. The property of acquiring a positive charge is indeed multiply realized with respect to a wide range of physical properties-simply let $\mathrm{R}$ be a set of physical properties which are sufficient, but not necessary, to bring about a positive charge (say, the set which includes only certain macro properties of an ignition system). Nothing of interest follows save 
that acquiring a positive charge is not identical to any physical property within this particular realization base.

On the other hand, the point is not entirely "fatuous," as Davidson claims. For let $\mathrm{R}$ range over all the physical properties, including the microphysical property of having a distribution of protons over electrons, and acquiring a positive charge is no longer multiply realized. That is, acquiring a positive charge is identical to having a distribution of protons over electrons, and so condition (b) in our definition of multiple realization is not satisfied.

Or consider the case of water. Even though the property of being water is identical with that of $\mathrm{H}_{2} \mathrm{O}$, and admittedly physical, it is nevertheless multiply realized with respect to many physical properties-again, restrict $\mathrm{R}$ to a set of properties which are sufficient, but not necessary, to insure the presence of water (say, certain atmospheric conditions, $\mathrm{H}_{2} \mathrm{O}$ excluded, which bring about humidity). It follows that the property of being water is not identical to any property within this particular realization base. But let $\mathrm{R}$ range over all the physical properties, including that of $\mathrm{H}_{2} \mathrm{O}$, and water is no longer multiply realized.

Consider now the case of a psychological property. What is interesting here is that, unlike the previous cases, a given psychological property can be multiply realized with respect to the entire range of physical properties! The realization base, in other words, may contain any of the properties of physical science, and this, precisely because no physical property provides both a necessary and sufficient condition for the instantiation of a psychological property, as the functionalist argument was intended to show.

So this is why we can accept the point that physical properties, like psychological properties, are multiply realized, while at the same time maintaining that psychological properties are not identical to physical properties. The reason is that they are multiply realized with respect to different sets of properties. The moral is that, from a philosophical point of view, what makes matters interesting is the range of the realization base.

How, then, does temperature stand with respect to the entire range of physical properties? As we have seen, temperature does not have a microphysical or molecular correlate, which makes it unlike water and positive charges. Nevertheless, the identification with the physical is straightforward. For temperature is not multiply realized with respect to all the physical properties because temperature is itself a property of physical science (thermodynamics), and no property is multiply realized with respect to itself.

Now this answer may strike one as worse than pedantic. After all, a psychological property is not multiply realized with respect to itself either, and so in both cases we can say that the property in question is multiply realized with respect to an indefinitely wide range of physical properties 
excluding itself. So how, on the basis of this similarity, can we draw a different conclusion about them vis-à-vis their status as a physical property? Or, given this similarity, why not count temperature as nonphysical too?

But the question betrays a confusion which I take underlies this entire line of response. The confusion is that the phenomenon of multiple realization should provide a criterion for what is to count as nonphysical. This, after all, was the whole point of drawing our attention to multiply realized physical properties. But to insist that multiple realization provide such a criterion, or that a defender of the argument must believe it to do so, is simply a mistake.

So my answer to the question: "How can we draw a different conclusion about them vis-à-vis their status as a physical property?" will be that other criteria are used to count something as a physical property, meaning here that it is a property of physical science. And more generally, then, since other criteria are used to place an item like temperature in the domain of physical science and assign others to psychology, this leaves muitiple realization to enter only afterward as a proof that the pre-categorized types are not identical.

Thus, the debate in the philosophy of psychology, taken up early on by the positivists, and more recently by Putnam and Fodor, concerned the identification of psychological properties with those of biology, chemistry, or physics. Clearly it was not multiple realization which was used to classify these properties into their respective categories. In the case of psychology, for example, the properties and states are typically thought to be explanatory of rational activity and involved in the etiology of purposeful behavior-criteria which are ill suited for the classification of a physical state, given that physical science seeks to explain phenomena which are not rational and which do not "behave" in the psychological sense at all.

It goes without saying, also, that since temperature does not explain rational activity, then, in spite of its being realized by various other physical states, it falls outside the category of a psychological property. Indeed, it is the different criteria I am vaguely referring to which determined the distinct sets of generalizations that we find within the sciences. In any case, the point I wish to make is that with the precategorized domains already in existence, multiple realization then provided a way of answering the questions which would naturally arise about inter-theoretic identity.

The same thing is true in the context of the mind/body debate, as it was carried out, for example, by dualists and central state materialists. There was a certain class of things like pains and thoughts which were grouped together under the heading of "the mental." What guided the choice of such items was certain special features which they alone enjoyed, like 
phenomenal quality (for the sensations), intentionality (for the propositional attitudes), and on some accounts a private or direct access had by the subject of those mental states (the introspective states). The question then arose as to whether these things could be reduced to others, more precisely, whether the mental types were identical to neurological, chemical, or basic physical types. What counted as "the physical," then, was again just the things which are included in the physical sciences. So, roughly, a property is mental if and only if it falls under intentional, phenomenal, or introspective state types, and a property is physical if and only if it falls under biological, chemical, or basic physical types. ${ }^{15}$

What these historical reminders serve to show are three important things. First, it is not true that a property is classified as being mental or nonphysical if and only if it is multiply realized (and hence it is a mistake to think that an item like temperature is mental or nonphysical just because it is multiply realized). For that we use other criteria. Second, and consequently, multiple realizability should not be saddled with the work of these other criteria. And third, the only work our multiple realizability argument can be expected to do is precisely to show that mental properties are not reducible to physical properties, all understood by their proper criteria.

Put differently, multiple realization by itself provides a test for identity, not nonphysicality; but being a test for identity, it is nevertheless adequate to show that psychological properties are not identical to physical properties. How is this accomplished? Again, by taking the precategorized properties in question, the psychological and the physical, and then applying the test of multiple realization. The result is that when it is applied to the entire range of physical properties, it is exceedingly probable that the test will come out negative, as far as identity and lawful coextensiveness are concerned.

\section{A Question About Intuitive Physical Properties}

There is, I think, one final matter which needs to be resolved. Granted, multiple realization may show that psychological properties are not identical to physical properties, where the latter are understood as the properties of physical science. All the same, perhaps this is too narrow a conception of the physical-witness other intuitive physical properties like that of being a table ${ }^{16}$ In other words, the property of being a table is not found within physical science either, though it is presumably physical in some other sense of the term. Hence, my arguments thus far leave open the possibility that psychological properties will count as physical in some intuitively broader sense as well.

Now I concede that this is true. Psychologically properties might count 
as physical in some intuitively broader sense. But there are three points which I think will considerably diminish whatever force this objection may have. First, as my brief historical remarks were intended to show, the conception of a physical property at play in the debate was that of being a property of physical science. The dispute in the philosophy of psychology, again, concerned the reduction of a human or special science to a physical or natural science (that is, the Geisteswissenschaften to the Naturwissenschaften); the traditional mind/body debate concerned the identity of phenomenal, intentional, and introspective state types with neurological, chemical, or basic physical types. Either way, it was physical science which circumscribed the legitimate bounds of the physical. Psychological properties, then, are irreducible vis-à-vis those properties, and nothing which appeals to a wider notion of the physical will detract from this point.

Second, we might try to deny the original datum which motivates this intuitively broader notion of the physical, whatever it may be, by denying that intuitive physical properties like a table are purely physical. How could this be? Perhaps because "being a table" cannot be specified without recourse to psychological notions, specifically, the intention that the object be used for certain purposes (a point which follows from the fact that there are no purely structural necessary and sufficient conditions for being a table). Now it might be thought that this point has a fairly limited application since there are other intuitive physical properties which do not involve an appeal to human intention, for example, being a rock, a tree, or even a planet. ${ }^{17}$ But notice that these are properties of physical science, of geology, biology, and astrophysics respectively; and hence they would count as physical on the criterion employed throughout, namely, being a property of physical science.

Of course, in response, one might question why psychological theory should not also count as one of these higher-level physical sciences. ${ }^{18}$ But our answer should now be clear. Psychological theory does not count as a physical science according to the traditional classification which was at work in the debate (it is a human or special science, not a physical or natural one; and, what lies behind this distinction, it deals with phenomenal, intentional, and introspective state types, as opposed to the other sciences). Moreover, its properties are not reducible to the properties of those physical sciences. Hence, to call psychology a "higherlevel physical science" would be a victory in name only. Nothing of substance would be gained, and all the original concerns would remain-how a human science relates to the others, how the intentional relates to the nonintentional, how a private phenomenal experience relates to the publicly accessible facts, and so on. Such concerns are not addresed by simply renaming psychology as a physical science.

Finally, my third point is that even if we do appeal to a broader and more intuitive notion of the physical, one which would include properties 
like being a table, and even if these other properties are not covertly psychological in the way suggested above, still, I doubt that psychological properties will count as physical on any intuitive scheme of classification. Why? Because it was precisely this kind of classification which was at work in the traditional mind/body debate. Specifically, it was an intuitive division of things based upon the prima facie difference between the intentional, the phenomenal, and the introspective, versus other things which do not have these features. Mental properties fall on one side. And the intuitive physical properties, like being a table, fall on the other. It therefore requires a philosophical theory to identify these intuitively distinct and separately classified items; or, as I have insisted upon, it requires the multiple realizability argument to show that they cannot be identified.

In conclusion, then, it seems that on our best and most interesting conception of what it is to be a physical property, the multiple realizability argument will suffice to show that psychological properties are not to be identified with physical properties. In particular, the mere fact that some physical properties are multiply realized by others is of no consequence. The mental and the physical will have distinct realization bases, and only in the case of the mental can a given property be realized with respect to the entire range of physical properties. ${ }^{19}$

University of Michigan Ann Arbor, Michigan

\section{NOTES}

${ }^{1}$ Hilary Putnam, "The Nature of Mental States," in Mind, Language and Reality (London: Cambridge University Press, 1975), pp. 429-440; and Jerry Fodor, "The Special Sciences (Or: The Disunity of Science as a Working Hypothesis)," in Representations (Cambridge Mass: M.I.T. Press, 1981), pp. 127-145.

${ }^{2}$ The criticism is found in Jaegwon Kim, "Phenomenal Properties, Psychophysical Laws, and the Identity Theory," Monist, vol. 56 (1972), pp. 190-191; Donald Davidson, "The Material Mind," in Essays on Actions and Events (Oxford University Press, 1980), pp. 251-252; and Mark Wilson, "What Is This Thing Called 'Pain'?- the Philosophy of Science Behind the Contemporary Debate," Pacific Philosophical Quarterly, vol. 66 (1985), pp. 227-267.

${ }^{3}$ Donald Davidson, "Mental Events," in Essays on Actions and Events, pp. 207-225; and "Psychology as Philosophy," ibid., pp. 229-244.

${ }^{4}$ Thomas Nagel seems to have argued in this way. See his "Physicalism," The Philosophical Review, vol. 74 (1965), pp. 351-352.

${ }^{5}$ Because functional theories are apt to raise the philosopher's brow, it should be noted that the present argument does not require any commitment to the controversial "psycho-functional identity theory." For the latter theory identifies commonsense or folk psychological properties with functional properties, and this may well be false given that commonsense states have not only "compositional" plasticity, as commonly argued, but 
also "computational" plasticity. See Putnam, Representation and Reality (Cambridge Mass: M.I.T. Press, 1988); and cp. Ned Block, "Troubles with Functionalism," in Block, ed., Readings in Philosophy of Psychology, vol. 1 (Cambridge Mass: Harvard University Press, 1980), pp. 291-293. Hence, our discussion must be restricted to the functional properties of psychology and not any common mental properties. All the same, our argument is not affected. If anything, it is enhanced. Common mental properties are multiply realized by functional states, and they in turn by diverse physical states, making type physicalism appear doubly mistaken. The mental is, as it were, twice removed from the physical.

${ }^{6}$ See Ned Block, "What Is Functionalism?," in Block, ed., Readings in Philosophy of Psychology, vol. 1, pp. 171-184; and Jerry Fodor, "Something of the State of the Art," in Representations, pp. 1-31.

${ }^{7}$ The appeal to infinite disjunctive properties is made by Jaegwon Kim in "Supervenience and Nomological Incommensurables," American Philosophical Quarterly, vol. 15 (1978), pp. 149-156; and "Concepts of Supervenience," Philosophy and Phenomenological Research, vol. 45 (1984), pp. 153-176. The appeal to species-specific properties is made by Kim in "Phenomenal Properties, Psychophysical Laws, and the Identity Theory," ibid., p. 190; David Lewis, "Mad Pain and Martian Pain," in Block, Readings in Philosophy of Psychology, vol. 1, pp. 219-220; and Berent Enc, "In Defense of the Identity Theory," Journal of Philosophy, vol. 80, (1983), pp. 289-291.

Species-specific properties have yet to be criticized in the literature, though I think it is clear that the proposal faces a number of difficulties, e.g., that multiple realization can occur within our species due to the plasticity of the brain. As for infinite disjunctive properties, I agree with the critics that such properties are ontologically objectionable since they do not do the work which properties are chiefly called upon to do, i.e., ground "objective resemblances" and contribute to an object's "causal powers." See D. M. Armstrong, A Theory of Universals (London: Cambridge University Press, 1978), esp. pp. 19-23; and Paul Teller, "Comments on Kim's Paper," Southern Journal of Philosophy, vol. 22 (1983), Supplement, pp. 57-61. Moreover, even granting infinite disjunctive physical properties, they will fail to reduce psychological properties on the assumption that it is nomically or at least metaphysically possible for a mental type to be realized in nonphysical systems-a possibility, it seems, which is not precluded by the nature of functional states. See Shoemaker, "Some Varieties of Functionalism," in J. I. Biro and Robert Shahan, eds., Mind, Brain, and Function (Norman: University of Oklahoma Press, 1982), p. 98; and cp. Putnam, "The Nature of Mental States," pp. 435-436.

${ }^{8}$ Kim, "Phenomenal Properties, Psychophysical Laws, and the Identity Theory," p. 191; see also "Supervenience and Nomological Incommensurables," p. 151.

9 "Phenomenal Properties, Psychophysical Laws, and the Identity Theory," p. 190; cp. also Ernest Nagel, The Structure of Science (New York: Harcourt Brace \& World Inc., 1961), p. 314.

10 "Phenomenal Properties, Psychophysical Laws, and the Identity Theory," p. 190.

${ }^{11}$ Davidson, "The Material Mind," pp. 251-252.

${ }^{12}$ Wilson, "What Is This Thing Called 'Pain'? - the Philosophy of Science Behind the Contemporary Debate," pp. 228-235. Aside from this, what is perhaps the main thrust of Wilson's paper is the claim that physics employs "extremely generous mathematical methods for constructing new traits" which will guarantee that all psychological properties have a lawfully coextensive physical property (ibid., p. 232). The argument, however, is not especially clear, and his examples appear to raise a number of different points. It suffices to say that if the physical property in question is indeed lawfully coextensive with the mental type, then either the physical system required to instantiate the property or the property itself would need to be wildly disjunctive (succumbing to all 
the concerns mentioned in $\mathrm{n} .7$ ). At least some such worry led one commentator to say that the system "must comprise most of the universe", and hence that the property in question would be physical in only a trivial and philosophically uninteresting sense. See Alan Nelson, "Physical Properties," Pacific Philosophical Quarterly, vol. 66 (1985), p. 276.

13 "What Is This Thing Called 'Pain'?- the Philosophy of Science Behind the Contemporary Debate," p. 228.

${ }^{14}$ Wilson, ibid, ; see also Enc, "In Defense of the Identity Theory," p. 289.

${ }^{15}$ I omit reference to other physical sciences, e.g., geology, astronomy, and astrophysics, simply because no one has proposed that mental properties be reduced to properties in their domain.

${ }^{16}$ I owe this point to Louis Loeb.

${ }^{17}$ Stephen Yablo brought this point to my attention.

${ }^{18}$ A question raised by an anonymous reader of this journal.

${ }^{19}$ I should like to thank Jaegwon Kim, Louis Loeb, William Taschek, and Stephen Yablo for their many helpful comments on this paper. 